\title{
On Some Class of Distance Functions for Measuring Portfolio Efficiency
}

\author{
Carlos Barros ${ }^{1}$, Walter Briec ${ }^{2}$, Hermann Ratsimbanierana ${ }^{2}$ \\ ${ }^{1}$ School of Economics and Management, Technical University of Lisbon, Lisbon, Portugal \\ ${ }^{2}$ Centre d'Analyse de l'Efficience et Performance en Économie et Management, University of Perpignan, \\ Perpignan, France \\ E-mail: cbarros@iseg.utl.pt, \{briec, hermann.ratsimbanierana\}@univ-perp.fr \\ Received May 19, 2011; revised July 22, 2011; accepted August 1, 2011
}

\begin{abstract}
Morey and Morey [1] have developed an approach for gauging portfolio efficiencies in the context of the Markowitz model. Following some recent contributions [2,3], this paper analyzes the axiomatic properties of distance functions extending an earlier approach proposed by Morey and Morey. The paper also focuses on the hyperbolic measure and the McFadden gauge function [4]. Among other things, overall, allocative and portfolio improvements possibilities (in term of return expansion or/and risk contraction) based upon the indirect mean-variance utility function are analyzed. Along this line, duality results are established in each case. This enables us to calculate the degree of risk aversion maximizing the investor indirect mean-variance utility function in either return expansion or risk contraction. An empirical illustration is provided and reveal ranking of preferred risks aversion for some "CAC40" assets.
\end{abstract}

Keywords: Distance Functions, Portfolio Selection, Efficient Frontier, Dualities, Risk Aversion

\section{Introduction}

Distance functions, have been introduced by Shephard to measure by Shephard [5] for efficiency measurement either in input or output orientation. At the same time, Markowitz [6,7] has formulated the mean-variance model, a mathematical approach for determining the optimal riskreturn trade-off for portfolio selection. This approach is based upon quadratic programming. However, its computational cost was very high. Hence, Sharpe $[8,9]$ had developed the simplified diagonal model and later formulated the capital asset pricing model (CAPM) with Lintner [10]. Markowitz [11] criticized the relation between risk and excess returns described by the linear model due to Sharpe and Lintner. He argued that different expected returns might surely be obtained from the same risk structure.

Nevertheless, the mean-variance approach is the cornerstone of portfolio management and risk assessment. The purpose of this paper is to provide a general taxonomy of ratio-based performance indicator for risk management. This contribution extends the analysis proposed by Morey and Morey [1] and also provides a new look at some more recent contributions. In [2,3] a general framework was introduced that is based upon the shortage function a concept introduced by Luenberger [12] in microeconomic analysis. Transposed in a portfolio optimization context, this function looks for possible simultaneous improvement of return and reduction of risk in the direction of a vector $\mathrm{g}$. Though this approach generalizes that of Morey and Morey in the mean-variance space, the choice of a direction remains much arbitrary. In this paper, we make other investigations about measures involving a proportionate improvement of risk and return. It is shown that the measure proposed by Morey and Morey satisfies a special type of duality, termed "fractional duality". It is also established that one can obtain a duality result linking the indirect mean-variance utility both to the hyperbolic measure and the McFadden gauge function [4]. Some mathematical programs are also proposed and we propose a procedure to measure the risk aversion from the Khum and Tucker multipliers.

Among other things we propose a procedure to compute the distance functions introduced by Morey and Morey in the case where short sales are allowed. Hence, it is then possible to measure the impact the budget constraint has on performance. This we do by introducing a special version of the Thomson metric. 
This paper is organized as follow. In Section 2, succinctly presents the basic tools of the portfolio management approach proposed in Briec et al. [2]. Section 3 focusses on the distance function proposed by Morey and Morey. We then study some of their more appealing properties. Duality results are analyzed in Section 4 and allow us to decompose efficiency following the Farrell approach [13]. Hence, the preferred risk aversion in input or output orientation can be computed. Sources of performances change are discussed in Section 5. Section 6 introduces an indicator based under the Thomson metric to measure the impact on the performance of managerial constraint. In the next section the dual properties of hyperbolic measures and McFadden gauge are analyzed. They are compared to the return oriented measure. The last section provides an empirical illustration with some "CAC40". A concludingsection outlines conclusions and possible extensions.

\section{Efficient Frontier and Portfolio Management}

This section introduces main ideas of the portfolio selection problem. Let us consider a market with $n$ financial assets. Note $E\left[R_{i}\right]$ for $i=1, \cdots, n$ the expected return of the asset $i$ and the covariance matrix of these assets such that $\Omega_{i, j}=\operatorname{Cov}\left[R_{i}, R_{j}\right]$ for $i, j \in\{1, \cdots, n\}$. A portfolio is an combination of one or more of these assets. Their proportions may be represented by the vector $x=$ $\left(x_{1}, x_{2}, \cdots, x_{n}\right)$ with $\sum_{i=1}^{n} x_{i}=1$ and $x_{i}>0$ if short sales is not allowed.

It is assumed throughout the paper that economic constraints (Pogues [14], Rudd and Rosenberg [15]) are linear functions of the asset weights. Thus, the set of the admissible portfolios may be written as follow :

$$
\mathfrak{I}_{A}=\left\{x=\mathbb{R}^{n}: \sum_{i=1}^{n} x_{i}=1, A x \geq 0\right\},
$$

where $A$ is an affine $m \times n$ map whose the range on $\mathbb{R}^{n}$ is a subset of $\mathbb{R}^{m}$. If Ax is null for all $x \in \mathbb{R}^{n}$ then we say that short-sales are allowed. In such a case, the set of admissible portfolios is extended from the unit simplex to an unbounded hyperplane. In general, the constraint $A x \geq 0$ represents the economic and managerial constraints the manager must deal with. The return of portfolio $x$ is $R(x)=\sum_{i=1}^{n} x_{i} R_{i}$. The expected return and its variance can be defined as follows: $E[R(x)]=\sum_{i=1}^{n} x_{i} E$ $\left[R_{i}\right]$ and $\operatorname{Var}[R(x)]=\sum_{i, j}^{n} x_{i} x_{j} \operatorname{Cov}\left[R_{i}, R_{j}\right]$, respectively. For the sake of simplicity, let us denote

$$
\mu(x)=E[R(x)] \text { and } \rho(x)=\operatorname{Var}[R(x)] .
$$

In addition, we consider the map $\Phi: \mathfrak{J}_{A} \rightarrow \mathbb{R}^{2}$ defined by

$$
\Phi(x)=(\rho(x), \mu(x))
$$

See for instance [20] for more details about meanvariance approaches and stochastic dominance. The return and the variance are continuous in $x$. Hence $\Phi\left(\mathfrak{J}_{A}\right)$ is a compact subset of $\mathbb{R}^{2}$. Following the Markowitz approach, the subset $\Phi\left(\mathfrak{J}_{A}\right)$ is important to identify the efficient portfolios. However, it is not convex and, consequently, this subset cannot be used for a quadratic programming approach. Briec, Lesourd and Kerstens [2] have extended the subset $\Phi\left(\mathfrak{I}_{A}\right)$ as follows:

$$
D R_{A}=\Phi(A)+\left(\mathbb{R}_{+} \times \mathbb{R}_{-}\right)
$$

It is important to notice that $D R_{A}$ is convex. Equivalently, one has:

$$
\begin{aligned}
D R_{A}=\{( & (r, m) \in \mathbb{R}^{2}: \\
& \left.\quad \exists x \in \mathfrak{I}_{A},(-r, m) \leq(-\rho(x), \mu(x))\right\}
\end{aligned}
$$

This set is not only compatible with the definition in Markowitz [6], it guarantees a minimum variance of the feasible portfolios analogously to a "Free-Disposal Hull". The subset of the all the mean-variance points that are not strictly dominated is termed the "efficient frontier". It is useful to define the efficient portfolios from the above definition.

Definition 2.1. The set of the weakly efficient portfolios $n$ the simplex is defined as:

$$
\begin{aligned}
\Lambda^{M}\left(\mathfrak{I}_{A}\right)=\{x \in \mathfrak{I}:(-\rho(x), \mu(x))<(-r, m) \\
\left.\Rightarrow(r, m) \notin D R_{A}\right\}
\end{aligned}
$$

For a given degree of risk aversion, Markowitz [6] defined the following utility function to compute the corresponding efficient portfolio.

$$
U_{\alpha, \beta}^{A}(x)=\beta \mu(x)-\alpha \rho(x)
$$

where $\alpha \geq 0$ and $\beta \geq 0$. The following program maximize this mean-variance utility function.

$$
\begin{gathered}
\max U_{\alpha, \beta}^{A}(x)=\beta \mu(x)-\alpha \rho(x) \\
\text { s.t. } A x \geq 0 \\
\sum_{i=1}^{n} x_{i}=1, x \geq 0
\end{gathered}
$$

where the ratio $\varphi=\alpha / \beta \in[0,+\infty]$ stands for risk aversion.

\section{Portfolio Efficiency Measures}

Measuring efficiency in a portfolio context accounts usually the possible return improvements and/or risk contractions. In this paper, we propose some class of measures which consider investor preferences for risk. 


\subsection{The Morey and Morey Distance Functions}

Two distance functions were introduced by Morey and Morey to gauge portfolio performance. The first function computes the maximum expansion of the mean of return for a given level of risk. The map $D_{R E}^{A}(x): \mathfrak{I} \rightarrow[1,+\infty]$ defined as:

$$
D_{R E}^{A}(x)=\sup \left\{\theta:(\rho(x), \theta \mu(x)) \in D R_{A}\right\}
$$

is called the Morey return expansion distance function. It is easy to see that this measure has some drawbacks for portfolios whose the return is not positive. Thus, we shall restrict its domain to the subset of $\mathfrak{I}$ defined by:

$$
\mathfrak{I}_{A}^{+}=\left\{x \in \mathfrak{I}_{A}: \mu(x)>0\right\} .
$$

Focusing on the risk contraction, the map $D_{R C}^{A}: \mathfrak{I} \rightarrow$ $[0,1]$ defined by:

$$
D_{R C}^{A}(x)=\inf \left\{\lambda:(\lambda \rho(x), \mu(x)) \in D R_{A}\right\}
$$

is called the Morey risk contraction distance function. Notice that this function may be zero valued when there is a riskless asset whose the variance is 0 . We propose some of their elementary properties which are essentials in portfolio performance gauging. To simplify the notations, we introduce the partial order $\succeq$ defined by:

$$
x \succeq y \Leftrightarrow(-\rho(x), \mu(x)) \geq(-\rho(y), \mu(y)) .
$$

Proposition 3.1. Let $D_{R E}^{A}$ be the mean expansion return distance defined in (3.1). $D_{R E}^{A}$ has the following properties:

i) $x \in \mathfrak{J}_{A}^{+} \Rightarrow 1 \leq D_{R E}^{A}(x) \leq+\infty$

ii) $D_{R C}^{A}(x)=1 \Leftrightarrow \Lambda^{M}\left(\mathfrak{I}_{A}\right)$ (Weak efficiency).

iii) $\forall x, y \in \mathfrak{I}$, if $x \succeq y$ then $D_{R E}^{A}(y) \succeq D_{R E}^{A}(x)$ (Weak monotonicity).

iv) $D_{R E}^{A}$ is continuous on $\mathfrak{J}_{A}$.

Proof. Let us prove $i$ ). The first inequality is immediate. From the definition of the representation set, if $x \in \mathfrak{I}_{+}$then the subset

$$
C(x)=\left\{(v, e) \in D R_{A}:(v,-e) \leq(\rho(x),-\mu(x))\right\}
$$

is bounded. It trivially follows that $D_{R E}(x)<+\infty$. To prove ii), assume that $x \notin \wedge^{M}\left(\mathfrak{J}_{A}\right)$. In such a case, there exists some $(v, e) \in D R_{A}$ such that $(-v, e) \leq$ $(-\rho(x), \mu(x))$. But, from Definition (3.1), it immediately follows that $D_{R E}^{A}(x)>1$. Consequently, it can be deduced that $D_{R E}^{A}(x)=1 \Rightarrow x \in \Lambda^{M}\left(\mathfrak{I}_{A}\right)$. To prove the converse, assume that $D_{R E}^{A}(x)>1$. We get:

$$
\left(-\rho(x), D_{R E}^{A}(x) \mu(x)\right) \leq(-\rho(x), \mu(x)) .
$$

It can immediately be deduced that $x \notin \wedge^{M}\left(\mathfrak{I}_{A}\right)$. Let us prove iii). $\forall x, y \in \mathfrak{I}_{A}$, if $\rho(y) \geq \rho(x)$ and $\mu(y)$ $\leq \mu(x)$ then $D_{R E}^{A}(y) \leq D_{R E}^{A}(x)$. From the notations above, we have $C(x) \subset C(y)$, for all $x, y \in \mathfrak{I}_{A}^{+}$. Consequently,

$\left\{\theta:(\rho(x), \theta \mu(x)) \in D R_{A}\right\} \subset\left\{\theta:(\rho(y), \theta \mu(y)) \in D R_{A}\right\}$,

and the result follows. We end by proving iv). Let $T: D R_{A} \rightarrow R_{+}$be the function defined by

$$
T(r, m)=\sup \left\{\theta:(r, \theta m) \in D R_{A}\right\}
$$

Since $D R_{A}$ is convex and satisfies the free disposal rule, it is easy to show the continuity of $T$ (see Shephard [5]). Hence $D_{R E}^{A}(x)$ is continuous on $\mathfrak{I}_{A}$.

The next result analyzes the case where the distance function involves a risk contraction of portfolios.

Proposition 3.2. Let $D_{R C}^{A}$ be the risk contraction distance function defined in (3.3). We have the following properties.

i) If there is no riskless asset then $0<D_{R C}^{A}(x) \leq 1$

ii) For all $x \in \mathfrak{I}_{A}, D_{R C}^{A}(x)=1 \Leftrightarrow D_{R E}^{A}(x)=1 \Leftrightarrow x \in$ $\wedge^{M}\left(\mathfrak{J}_{A}\right) \quad$ (Weak efficiency)

iii) $\forall x, y \in \mathfrak{J}_{A}, \quad x \succeq y \Leftrightarrow D_{R C}^{A}(y) \geq D_{R C}^{A}(x) \quad$ (Weak monotinicity).

iv) $D_{R C}^{A}$ is continuous on $\mathfrak{J}_{A}$.

Proof. With obvious changes, the proofs are similar to those of Proposition (3.1).

\subsection{The Efficiency Improvement Possibility Function}

To gauge portfolio efficiency, Briec, Lesourd and Kerstens [2] have introduced a variation of the shortage function which computes simultaneously risk reduction and expected return improvement. For a portfolio $x$ in $D R$, the direction of the shortage function is determined by the vector $g=\left(-g_{V}, g_{E}\right)$. Formally, this efficiency improvement possibility function is defined by:

$$
S_{g}(x)=\sup \left\{\delta ;\left(\rho(x)-\delta \mathrm{g}_{V}, \mu(x)+\delta \mathrm{g}_{E}\right) \in D R\right\}
$$

The following quadratic program computes the maximum percentage improvement of the portfolio $y_{k}$ :

$$
\begin{aligned}
& \max \delta \\
& \text { s.t. } \mu\left(y_{k}\right)+\delta g_{E} \leq \sum_{i=1}^{n} x_{i} E\left[R_{i}\right] \\
& \rho\left(y_{k}\right)-\delta g_{V} \geq \sum_{i=1}^{n} x_{i} V\left[R_{i}\right] \\
& A x \leq b, \sum_{i=1 \cdots n} x_{i}=1, x_{i} \geq 0, i=1 \cdots n
\end{aligned}
$$

For more details of the basic properties about this function, see for instance Briec et al. [2].

\subsection{Hyperbolic and McFadden Distance Function}

We introduce two distance functions defined in the mean- 
variance graph. We analyze the basic properties and establish a duality result. Measuring efficiency in a portfolio context accounts the possible return improvements and/or risk contractions. In the following, we introduce two specific measures. The first one, called hyperbolic distance function, computes the maximum simultaneous shrinkage and expansion of the risk and expected return respectively. It is defined as:

$$
D_{H}^{A}(x)=\sup \left\{\theta:\left(\frac{1}{\theta} \rho(x), \theta \mu(x)\right) \in D R_{A}\right\}
$$

We also introduce the McFadden gauge that computes the maximum proportionate expansion of the risk and expected return respectively:

$$
G_{M F}^{A}(x)=\sup \left\{\theta:(\theta \rho(x), \theta \mu(x)) \in D R_{A}\right\}
$$

We propose some of their elementary properties which are essentials in portfolio performance gauging. Figure 1 illustrate the basic ideas behind the definitions above. The "hyperbolic distance function" simultaneously involves a contraction of the risk and an expansion of the expected return. The "McFadden gauge function" is very different because it computes simultaneously the maximum expansion of the risk and the expected return of investors.

Proposition 3.3. The map $D_{H}^{A}$ defined in (3.1) has the following properties:

i) $x \in \mathfrak{I} \Rightarrow D_{H}^{A}(x)<+\infty$

ii) if $\mu(x) \geq 0$, then $D_{H}^{A}(x)=1 \Leftrightarrow x \in \partial^{M}\left(\mathfrak{I}_{A}\right)$ (Weak efficiency).

iii) $\forall x, y \in \mathfrak{I}$, if $x \succeq y$ then $D_{H}^{A}(y) \leq D_{H}^{A}(x)$ (Weak monotonicity).

iv) $D_{H}^{A}$ is continuous on $\mathfrak{I}_{A}$.

Proof. The proofs are very similar to those concerning the maximum risk expansion distance function and thus it is omitted.

The mathematical program, one need to solve is the following:

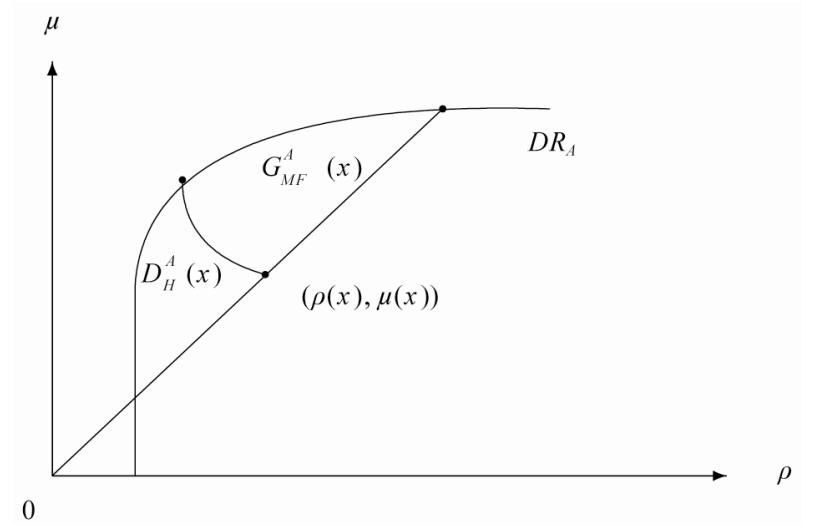

Figure 1. Hyperbolic Distance Function and McFadden Gauge.

$$
\begin{gathered}
D_{H}^{A}(y)=\max \lambda \\
\text { s.t. } \theta \mu(y) \leq \sum_{i=1}^{n} x_{i} E\left[R_{i}\right] \\
\frac{1}{\theta} \rho(y) \geq \sum_{i, j=1}^{n} \Omega_{i, j} x_{i} x_{j} \\
\sum_{i=1}^{n} x_{i}=1, A x \geq 0 .
\end{gathered}
$$

The next result analyzes the case where the distance function involves a risk contraction of portfolios. In the following we denote $\left(G_{M F}^{A}\right)=\left\{x \in \mathfrak{J}_{A}: G_{M F}^{A}<+\infty\right\}$.

Proposition 3.4. Let $G_{M F}^{A}$ be the map defined in (3.3). We have the following properties.
i) If $\mu(x)>0$ then $G_{M F}^{A}(x)<+\infty$.
ii) $G_{M F}^{A}(x)=1 \Rightarrow x \in \partial^{M}\left(\mathfrak{I}_{A}\right) \quad$ (Weak efficiency)
iii) $\forall x, y \in \mathfrak{I}_{A}$,
$(\rho(y), \mu(y)) \leq(\rho(x), \mu(x)) \Leftrightarrow G_{M F}^{A}(y) \leq G_{M F}^{A}(x)$.

Proof. i) Since $\mathfrak{I}_{A}$ is a compact set it follows that $\Phi\left(\mathfrak{I}_{A}\right)$ is a compact subset of $\mathbb{R}^{2}$. Therefore it is norm bounded. Consequently, by definition, $D R_{A}$ is bounded in its return dimension. Thus $G_{M F}^{A}(x)<+\infty$. ii) Clearly, if $G_{M F}^{A}(x)=1$ then the point $(\rho(x), \mu(x))$ lies on the upper part of the mean-variance frontier. However, all these frontier points are efficient which ends the proof. The proof of iii) is immediate.

Notice that, in general, the gauge function is not continuous on $\mathfrak{J}_{A}$. Moreover, it does not characterize the weak efficient frontier. The next result offers a comparison between all the distance functions introduced in the paper.

Lemma 3.5. For all $x \in \mathfrak{I}_{A}$ and such that $\mu(x)>0$, we have: $D_{H}^{A}(x) \leq D_{R E}^{A}(x) \leq G_{M F}^{A}(x)$.

Proof. Clearly, we have the inclusion

$\left\{\left(\theta: \frac{1}{\theta} \rho(x), \theta \mu(x)\right) \in D R_{A}\right\} \subset\left\{\theta:(\rho(x), \theta \mu(x)) \in D R_{A}\right\}$, hence we obtain the first inequality. To prove the second one, fix $\bar{\theta}=D_{R E}^{A}(x)$. By definition $(\rho(x), \bar{\theta} \mu(x)) \in D R_{A}$. However, by construction $D R_{A}=D R_{A}+\left(\mathbb{R}_{+} \times \mathbb{R}_{-}\right)$. Since $\bar{\theta} \geq 1$, we then deduce that $(\bar{\theta} \rho(x), \theta \mu(x)) \in D R_{A}$. Thus, $G_{M F}^{A} \quad(x) \geq \bar{\theta} \quad$ which ends the proof.

Suppose that $\mu(y)>0$, the mathematical program one should compute is the following:

$$
\begin{gathered}
G_{M F}^{A}(y)=\max \theta \\
\text { s.t. } \theta \mu(y) \leq \sum_{i=1}^{n} x_{i} E\left[R_{i}\right] \\
\theta \rho(y) \geq \sum_{i, j=1}^{n} \Omega_{i, j} x_{i} x_{j} \quad\left(P_{M F}\right) \\
\sum_{i=1}^{n} x_{i}=1, A x \geq 0 .
\end{gathered}
$$




\section{Duality and Graph Distance Functions}

Following our earlier results, we establish a link between these distance functions and the indirect mean-variance utility function, except for the shortage function which has already been discussed in [2]. The indirect meanvariance utility gives the portfolio which achieves a maximal utility of an agent given his (her) risk aversion. Thus, fixing some parameters $\alpha, \beta$, it is well known that an efficient portfolio maximizing the utility can be calculated using standard procedures of quadratic optimization. The mean-variance indirect utility function is defined by $V^{A}: \mathbb{R}_{+}^{2} \rightarrow \mathbb{R}$ by:

$$
V^{A}(\alpha, \beta)=\sup \left\{\beta \mu(x)-\alpha \rho(x): x \in \mathfrak{I}_{A}\right\}
$$

This function associates to the pair $(\rho, \mu)$, which stands for the risk aversion, the maximal utility level a fund manager can expect.

\subsection{Morey and Morey Fractional Duality}

Morey and Morey's approach allows to distinguish two dual relations with the indirect mean-variance utility functions. They are expressed in term of return expansion and risk contraction respectively. We show that the distance functions we have introduced can be related to the maximisation of this utility functions with an optimal degree of risk aversion. Following Briec, Kerstens and Lesourd [2], duality results allows a decomposition of efficiency measures. This is done paralleling an earlier concept due to Farrell [13] in a production theory context. However, in view of the nature of the tools they used, these duality results have an additive structure, while the measure proposed by Morey and Morey has a multiplicative one. In this subsection, we shall prove that the distance functions proposed by Morey and Morey also have a dual interpretation based upon the indirect meanvariance utility function.

\subsubsection{Efficiency Decompositions}

Given portfolio, the overall return expansion is the ratio computed as its maximum return by its return value independently of the asset price information. Suppose that $\mu(x)>0$, i.e. $x \in \mathfrak{J}_{A}^{+}$. The overall return expansion $\left(O_{R E}\right)$ index is then defined as the quantity:

$$
\begin{gathered}
O_{R E}(x, \alpha, \beta) \\
=\sup \left\{\theta: \theta \beta \mu(x)-\alpha \rho(x) \leq V^{A}(\alpha, \beta)\right\} .
\end{gathered}
$$

Hence, one can equivalently write

$$
O_{R E}(x, \alpha, \beta)=\frac{V^{A}(\alpha, \beta)+\alpha \rho(x)}{\beta \mu(x)}
$$

The allocative return expansion $A_{R E}$ index corres- ponds to the portfolio reallocation required to achieve the maximum of the the indirect mean-variance utility. It is defined as follow:

$$
A_{R E}(x, \alpha, \beta)=O_{R E}(x, \alpha, \beta) / D_{R E}^{A}(x)
$$

The portfolio return expansion $\left(P_{R E}\right)$ index $t$ is the quantity:

$$
P_{R E}(x)=D_{R E}^{A}(x) .
$$

The Farrell decomposition is then, by definition:

$$
O_{R E}(x, \alpha, \beta)=A_{R E}(x, \alpha, \beta) \cdot P_{R E}(x) .
$$

Using a symmetrical approach, one can introduce an overall risk contraction $\left(O_{R C}\right)$ index, an allocative risk contraction $\left(A_{R E}\right)$ index and a portfolio risk contraction $\left(P_{R C}\right)$ index. The overall risk contraction $\left(O_{R C}\right)$ index defined as follows:

$$
\begin{aligned}
& O_{R C}(x, \alpha, \beta) \\
& =\inf \left\{\lambda: \beta \mu(x)-(\lambda \alpha) \rho(x) \leq V^{A}(\alpha, \beta)\right\} .
\end{aligned}
$$

Equivalently, one has:

$$
O_{R C}(x, \alpha, \beta)=\frac{\beta \mu(x)-V^{A}(\alpha, \beta)}{\alpha \rho(x)}
$$

The allocative risk contraction index is

$$
A_{R C}(x, \alpha, \beta)=O_{R C}(x, \alpha, \beta) / D_{R C}^{A}(x),
$$

and the portfolio risk-contraction index is:

$$
P_{R C}(x)=D_{R C}^{A}(x) .
$$

By definition, we have:

$$
O_{R C}(x, \rho, \mu)=A_{R C}(x, \alpha, \beta) \cdot P_{R C}(x)
$$

If the overall risk contraction $O_{R C}=P_{R C}=1$ then we have an efficient portfolio and the allocative risk contraction $A_{R C}=1$ is certainly equal to one.

\subsubsection{Implicit Risk Aversion}

Duality between indirect utility functions and Morey and Morey's distance functions involves an implicit risk aversion which makes the selected portfolio optimal regarding to the mean-variance utility function.

Allocative return expansion may change with respect to the risks aversion parameter. Given portfolio, one can calculate by how much the level of $\phi=\rho / \mu$ needs to be increased to optimize overall portfolio efficiency. The earlier decompositions show that Allocative return expansion is greater than zero excepted whenever the selected portfolio lies on the indifference curve of the mean-variance utility function at its optimal level. Hence, if $O_{M R E}=\theta^{*}$, we have:

$$
V^{A}(\alpha, \beta)=\beta \theta^{*} \mu(x)-\alpha \rho(x)
$$


It follows that:

$$
\theta^{*}=\frac{V^{A}(\alpha, \beta)+\alpha \rho(x)}{\beta \mu(x)}
$$

It follows that $\theta^{*} \geq D_{R E}^{A}(x)$. Hence, we have:

$$
D_{R E}^{A}(x) \leq \frac{V^{A}(\alpha, \beta)+\alpha \rho(x)}{\beta \mu(x)} .
$$

Proposition 4.1. For all portfolio $x \in \mathfrak{I}_{A}$ such that $\mu(x)>0$, we have

$$
D_{R E}(x)=\inf \left\{\frac{V^{A}(\alpha, \beta)+\alpha \rho(x)}{\beta \mu(x)}:(\alpha, \beta) \geq 0\right\} .
$$

Proof. From its definition, the representation set that is convex, then it is the intersection of all its supporting hyperplanes (see [2]). Hence, we have:

$$
D R_{A}=\bigcap_{(\alpha, \beta) \geq 0}\left\{(v, e) \in \mathbb{R}^{2}: \beta e-\alpha v \leq V^{A}(\alpha, \beta)\right\} .
$$

We can equivalently write

$$
D_{R E}^{A}(x)=\inf \left\{\theta:(\rho(x), \theta \mu(x)) \notin D R_{A}\right\} .
$$

Let us denote $H(\alpha, \beta)=\left\{(v, e) \in \mathbb{R}^{2}: \beta e-\alpha v \leq V^{A}\right.$ $(\alpha, \beta)\}$ for all $(\alpha, \beta) \geq 0$. It follows that

$$
D_{R E}^{A}(x)=\inf \left\{\theta:(\rho(x), \theta \mu(x)) \in \mathbb{R}^{2} \backslash \bigcap_{(\alpha, \beta) \geq 0} H(\alpha, \beta)\right\} \text {. }
$$

Since

$$
\inf \{\theta:(\rho(x), \theta \mu(x)) \in H(\alpha, \beta)\}=\frac{V^{A}(\alpha, \beta)+\alpha \rho(x)}{b \mu(x)},
$$

we immediately deduce the result.

Paralleling the approach above, one can consider the situation where the risk of a given portfolio is contracted while fixing the returns at an arbitrary level. Following, Farrell decompositions, Allocative risk contraction is equal to one if a portfolio maximizes the mean varianceutility function. In such a case the Overall risk contraction is equal to one. Hence, in general, if we have $\lambda^{*}=O_{R C}$, we have

$$
\begin{aligned}
V^{A}(\alpha, \beta) & =\beta \mu(x)-\alpha \lambda^{*} \rho(x) \\
& \Leftrightarrow \lambda^{*}=\frac{\beta \mu(x)-V^{A}(\alpha, \beta)}{\alpha \rho(x)} .
\end{aligned}
$$

Therefore, we deduce that

$$
D_{R C}^{A}(x) \geq \frac{\beta \mu(x)-V^{A}(\alpha, \beta)}{\alpha \rho(x)}
$$

In the next result, we show that one can go a bit further by establishing the following duality result:

Proposition 4.2. For all portfolio $x \in \mathfrak{J}_{A}$, we have

$$
D_{R C}^{A}(x)=\sup \left\{\frac{\beta \mu(x)-V^{A}(\alpha, \beta)}{\alpha \rho(x)}:(\alpha, \beta) \geq 0\right\} \text {. }
$$

Proof. From the definition of the representation set we have: $D R_{A}=\bigcap_{(\alpha, \beta) \geq 0}\left\{(v, e) \in \mathbb{R}^{2}: \beta e-\alpha v \leq V^{A}(\alpha, \beta)\right\}$. We can equivalently write

$$
D_{R C}^{A}(x)=\sup \left\{\lambda:(\lambda \rho(x), \mu(x)) \notin D R_{A}\right\} .
$$

It follows that

$$
D_{R C}^{A}(x)=\sup \left\{\lambda:(\rho(x), \theta \mu(x)) \in \mathbb{R}^{2} \backslash \bigcap_{(\alpha, \beta) \geq 0} H(\alpha, \beta)\right\},
$$

where $H(\alpha, \beta)=\left\{(v, e) \in \mathbb{R}^{2}: \beta e-\alpha v \leq V^{A}(\alpha, \beta)\right\}$ for all $(\alpha, \beta) \geq 0$. Since

$$
\sup \{\lambda:(\lambda \rho(x), \mu(x)) \in H(\alpha, \beta)\}=\frac{\beta \mu(x)-V^{A}(\alpha, \beta)}{\alpha \rho(x)},
$$

we immediately deduce the result.

\subsubsection{Computing the Implicit Risk Aversion Degree}

In this subsection we show how to compute the implicit risk aversion. First, we define the mathematical programs one should deal with to compute the distance functions. Notice that though these programs have some analogies to those proposed in [16] and its subsequent development, they are not non-parametric $[17,18]$. In fact the frontier has a quadratic functional representation and, consequently the model is parametric. An illustration is proposed in $[19,20]$, where a stochastic frontier approach is proposed. However, the piecewise approximation obtained from the projection onto the frontier is a nonparametric estimation of the disposal representation set (see [2]). The following programs where first proposed by [1]. In the risk-oriented case we have, for all portfolio $y:$

$$
\begin{gathered}
D_{R C}^{A}(y)=\min \lambda \\
\text { s.t. } \mu(y) \leq \sum_{i=1}^{n} x_{i} E\left[R_{i}\right] \\
\lambda \rho(y) \geq \sum_{i, j=1}^{n} \Omega_{i, j} x_{i} x_{j} \quad\left(P_{R C}\right) \\
\sum_{i=1}^{n} x_{i}=1, A x \geq 0 .
\end{gathered}
$$

In the return-oriented case, if $\mu(y)>0$, we have:

$$
\begin{gathered}
D_{R E}^{A}(y)=\max \theta \\
\text { s.t. } \theta \mu(y) \leq \sum_{i=1}^{n} x_{i} E\left[R_{i}\right] \\
\rho(y) \geq \sum_{i, j=1}^{n} \Omega_{i, j} x_{i} x_{j} \\
\sum_{i=1}^{n} x_{i}=1, A x \geq 0 .
\end{gathered}
$$

It will be proven in the remainder that the implicit risk 
aversion can be deduced from the Kuhn-Tucker multipliers of the above programs. Two set valued maps taking into account either a risk-contraction or a return-expansion distance function are now introduced.

Definition 4.3. The set-valued map $\gamma: \mathfrak{I}_{A} \rightarrow 2^{\mathbb{R}_{+}^{2}}$ defined by

$$
\gamma_{R C}(x)=\operatorname{argmax}\left\{\frac{\beta \mu(x)-V^{A}(\alpha, \beta)}{\beta \rho(x)}:(\alpha, \beta) \geq 0\right\}
$$

is called the adjusted risk-contraction function. The setvalued map $\gamma: \mathfrak{J}_{A}^{+} \rightarrow 2^{\mathbb{R}_{+}^{2}}$ defined by

$$
\gamma_{R E}(x)=\operatorname{argmin}\left\{\frac{V^{A}(\alpha, \beta)+\alpha \rho(x)}{\beta \mu(x)}:(\alpha, \beta) \geq 0\right\}
$$

is called the adjusted return-expansion function.

Notice that, in the return oriented case, we have limited our definition to $\mathfrak{J}_{A}^{+}$that is a portfolio subset of $\mathfrak{I}$. These definitions allow to provide a formal definition of the implicit risk aversion.

Definition 4.4. Suppose that the maps $\varphi_{R C}$ and $\varphi_{R E}$ are single-valued at $x$. The implicit risk-aversion degree in the risk oriented and return oriented case are defined respectively by:

$$
R A_{R C}(x)=\frac{\gamma_{R C, 1}(x)}{\gamma_{R C, 2}(x)} \text { and } R A_{R E}(x)=\frac{\gamma_{R E, 1}(x)}{\gamma_{R E, 2}(x)} .
$$

In the case where $\varphi_{R C}$ and $\varphi_{R E}$ are not singlevalued at $x$, we say the the risk aversion is undefined.

The next result shows that the Kunh-Tucker Multipliers of the mathematical programs above can be used to find the implicit risk aversion.

Proposition 4.5. Suppose that the maps $\gamma_{R C}$ is singlevalued at $x$. Let $\left(\alpha_{R C}, \beta_{R C}\right)$ denotes the Kuhn-Tucker multipliers of the first and second constraints of Program $\left(P_{R C}\right)$, respectively. We have

$$
\gamma_{R C}(x)=\left(\alpha_{R C}, \beta_{R C}\right) \text { and } R A_{R C}(x)=\frac{\alpha_{R C}}{\beta_{R C}} .
$$

Proof. To prove this result, we use an earlier result due to Briec et al. [2] who used the shortage function $S_{g}^{A}$ that is defined for a given portfolio $x$ by:

$$
S_{g}^{A}(x)=\sup \left\{\delta:\left(\rho(x)-\delta g_{v}, \mu(x)+\delta g_{e}\right) \in D R_{A}\right\}
$$

Setting, $g_{v}=\rho(x)$ and $g_{e}=0$, we obtain:

$$
S_{g}^{A}(x)=1-D_{R C}^{A}(x) .
$$

However, from [2], we have:

$$
S_{g}^{A}(x)=\inf _{(\alpha, \beta) \ngtr 0}\left\{\frac{V^{A}(\alpha, \beta)-\beta \mu(x)+\alpha \rho(x)}{\alpha g_{v}+\beta g_{e}}\right\} .
$$

Since $g_{v}=\rho(x)$ and $g_{e}=0$, we have

$$
S_{g}^{A}(x)=\inf _{(\rho, \mu) \supsetneqq 0}\left\{\frac{V^{A}(\alpha, \beta)-\beta \mu(x)+\alpha \rho(x)}{\alpha \rho(x)}\right\} .
$$

Making an elementary transformation, we obtain:

$$
S_{g}^{A}(x)=1-\sup \left\{\frac{\beta \mu(x)-V^{A}(\alpha, \beta)}{\alpha \rho(x)}:(\alpha, \beta) \geq 0\right\} .
$$

Hence, from Proposition 4.2 the solution of the dual optimization program in (4.17) is identical to $\gamma_{R C}(x)$. However, from [2], this solution coïncides with the Kuhn and Tucker multiplier. If the dual solution is unique the result follows.

Proposition 4.6. Suppose that the maps $\gamma_{R E}$ is singlevalued at $x \in \mathfrak{I}_{A}^{+}$. Let $\left(\alpha_{R E}, \beta_{R E}\right)$ denotes the KuhnTucker multipliers of the first and second constraints of Program $\left(P_{R E}\right)$, respectively. We have

$$
\gamma_{R E}(x)=\left(\alpha_{R E}, \beta_{R E}\right), \text { and } R A_{R E}(x)=\frac{\alpha_{R E}}{\beta_{R E}} .
$$

Proof. The proof is similar to that of Proposition using [2] and setting, $g_{v}=0$ and $g_{e}=\mu(x)$.

\subsection{Hyperbolic and McFadden Distance Functions and Duality Result}

We can also establish duality between these distance functions and the indirect mean-variance utility function.

Proposition 4.7. For all portfolio $x \in \mathfrak{I}_{A}$ such that $\mu(x)>0$, we have

$$
\begin{aligned}
& D_{H}^{A}(x)= \\
& \inf _{(\alpha, \beta) \geq 0}\left\{\frac{\left.\left[V^{A}(\alpha, \beta)^{2}+4 \alpha \beta \mu(x) \rho(x)\right]\right]^{1 / 2}-V^{A}(\alpha, \beta)}{2 \beta \mu(x)}\right\} .
\end{aligned}
$$

Proof. From the definition of the representation set we have:

$$
D R_{A}=\bigcap_{(v, e) \geq 0}\left\{(v, e) \in \mathbb{R}^{2}: \beta e-\alpha v \leq V^{A}(\alpha, \beta)\right\} .
$$

We can equivalently write

$$
D_{H}^{A}(x)=\inf \left\{\theta:\left(\frac{1}{\theta} \rho(x), \theta \mu(x)\right) \notin D R_{A}\right\} .
$$

Let us denote $H(\alpha, \beta)=\left\{(v, e) \in \mathbb{R}^{2}: \beta e-\alpha v \leq V^{A}\right.$ $(\alpha, \beta)\}$ for all $(\alpha, \beta) \geq 0$. It follows that

$$
D_{H}^{A}(x)=\inf \left\{\theta:\left(\frac{1}{\theta} \rho(x), \theta \mu(x)\right) \in \mathbb{R}^{2} \backslash \bigcap_{(\rho, \mu) \geq 0} H(\alpha, \beta)\right\} .
$$

Solving a second order equation yields:

$$
\begin{gathered}
\inf \left\{\theta:\left(\frac{1}{\theta} \rho(x), \theta \mu(x)\right) \in \mathbb{R}^{2} \backslash H(\alpha, \beta)\right\} \\
=\frac{\left[V^{A}(\alpha, \beta)^{2}+4 \beta \alpha \mu(x) \rho(x)\right]^{1 / 2}-V^{A}(\alpha, \beta)}{2 \beta \mu(x)} .
\end{gathered}
$$


Since $\mathbb{R}^{2} \backslash \bigcap_{(\alpha, \beta) \geq 0} H(\alpha, \beta)=\bigcup_{(\alpha, \beta) \geq 0} \mathbb{R}^{2} \backslash H(\alpha, \beta)$, we immediately deduce the result.

A simpler duality result can be established regarding to the McFadden Gauge function [4].

Proposition 4.8. For all portfolio $x$ such that $\mu(x)$ $>0$, we have

$$
G_{M F}^{A}(x)=\inf _{(\alpha, \beta) \geq 0} \frac{V^{A}(\alpha, \beta)}{U_{\alpha, \beta}^{A}(x)} .
$$

Proof. The result is immediate using the fact that, if $x \in \operatorname{dom}\left(G_{M F}^{A}\right)$, there is at least some halfspace $H(\rho$, $\mu$ ) such that

$$
\inf \left\{\theta:(\theta \rho(x), \theta \mu(x)) \in \mathbb{R}^{2} \backslash H(\alpha, \beta)\right\}=\frac{V^{A}(\alpha, \beta)}{U_{\alpha, \beta}^{A}(x)} .
$$

\section{Impact of Managerial Constraints on Portfolio Selection and Short Sales}

This section analyzes how performance measurement varies regarding to the managerial constraints summarized by the constraint $A x \geq 0$. First, notice that if $A x=0$ for all $x \in \mathbb{R}^{n}$, then the set of portfolio is defined by

$$
\mathfrak{J}_{0}=\left\{x \in \mathbb{R}^{n}: \sum_{i=1}^{n} x_{i}=1\right\} .
$$

Since in such a case the map $A$ is identically null, we replace the subscript " $A$ ", with " 0 ". Suppose $B$ is an affine map defined on $\mathbb{R}^{n}$. Let $\mathcal{A}$ be the set of all the affine maps defined on $\mathfrak{I}_{A}$. In the following, for all $A, B \in \mathcal{A}$, we denote $A \geq B$ if $A x \geq B x$ for all $x \in$ $\mathbb{R}^{n}$. Next, we introduce a specific measure inspired from the Thomson metric that has some similarities to the well known Hilbert projective metric.

Definition 5.1. The map $\mathcal{M}_{R C}: \mathcal{A} \times \mathcal{A} \rightarrow[1,+\infty]$ defined by

$$
\begin{gathered}
\mathcal{M}_{R C}(A, B)=\sup _{x \in \mathfrak{I}_{A} \cap \mathfrak{I}_{B}} d_{x}(A, B) \text { where } \\
d_{x}(A, B)=\max \left\{\frac{D_{R C}^{A}(x)}{D_{R C}^{B}(x)}, \frac{D_{R C}^{B}(x)}{D_{R C}^{A}(x)}\right\}
\end{gathered}
$$

is called the risk oriented distance between $A$ and $B$.

Typically, $d_{x}$ is inspired from the Thomson metric, because it computes from a radial projection the maximum difference at $a$ between $A$ and $B$. The main difference comes from the fact that the Thomson metric computes the distance between two points. Hence, $d_{x}$ allows to define the map $\mathcal{M}$ that measures some kind of distance between two sets of managerial constraints. By virtue of its nature, this index takes values greater than one for portfolios whose the efficiency score is affected by the managerial constraints. If the metric is equal to one for each portfolio of a sample, then the efficiency scores are not affected by the managerial constraints. However, this does not mean that the efficient frontiers respectively obtained from $A$ and $B$ is identical. An interesting case arise in the situation where $A$ is the identity map (that is $A=I$ ) and $B$ is identically null. In such a case this measure allows to compare the situations with and without short sale and we not $B=0$. The following properties are trivial:

Lemma 5.2. Suppose that $\mathfrak{I}_{A} \cap \mathfrak{I}_{B} \neq \varnothing$.

i) If $B \geq A$ then

$$
\mathcal{M}_{R C}(A, B)=\inf _{x \in \mathfrak{I}_{A} \cap \mathfrak{I}_{B}} \max \left\{\frac{D_{R C}^{A}(x)}{D_{R C}^{B}(x)}\right\}
$$

ii) If $A=B$ then $\mathcal{M}_{R C}(A, B)=1$.

iii) If $\mathcal{M}_{R C}(A, B)>1$ then $A \neq B$.

Figure 2 depicts the idea the measure is based on. In line with $[14,15]$, the efficient frontier is modified according to the managerial constraints an investor is dealing with. Hence, the performance of portfolios is also modified by shift of the frontier. In particular, this measure is useful to test the impact of short sales impact on the performance of a given portfolio $x$. In such a case, we have:

$$
d_{x}(I, 0)=\max \left\{\frac{D_{R C}^{I}(x)}{D_{R C}^{0}(x)}, \frac{D_{R C}^{0}(x)}{D_{R C}^{I}(x)}\right\},
$$

and

$$
\mathcal{M}_{R C}(I, 0)=\inf _{x \in \mathfrak{I}_{I} \cap \mathfrak{I}_{0}} d_{x}(I, 0) .
$$

Since, $\mathfrak{I}_{I} \subset \mathfrak{I}_{0}$, we have

$$
\mathcal{M}_{R C}(I, 0)=\sup _{x \in \widetilde{I}_{I}}(I, 0) .
$$

If there exists some portfolio $y \in \mathfrak{I}_{0}$ such that $\mu(y)$ $=\mu(x)$ and $\rho(y)<D_{R C}^{I}(x) \rho(x)$ then it is easy to see that $\mathcal{M}_{R C}(I, 0)>1$. Hence, in this situation, the possibility of short sales enables decision makers to find a risk-

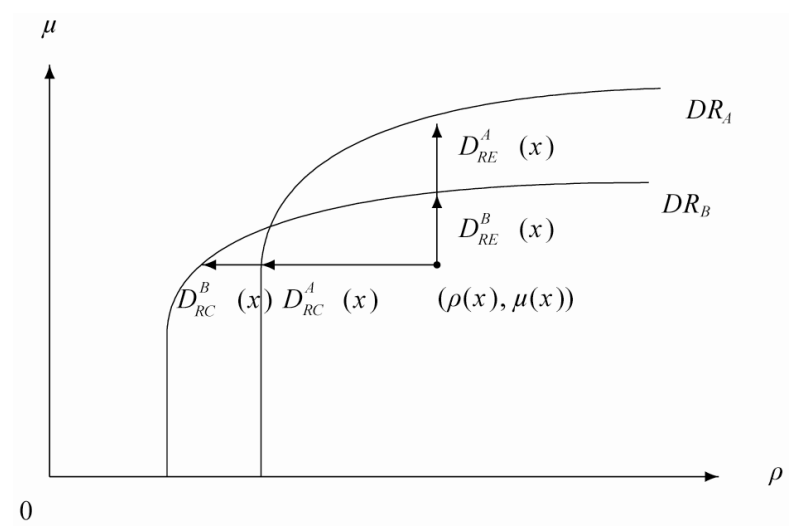

Figure 2. Impact of Managerial Constraints. 
less portfolio. A similar approach is possible when one looks for possible improvement of the return.

Definition 5.3. The map $\mathcal{M}_{R C}: \mathcal{A} \times \mathcal{A} \rightarrow[1,+\infty]$ defined by

$$
\begin{aligned}
\mathcal{M}_{R E}(A, B) & =\sup _{x \in \Im_{A}^{+} \cap \Im_{B}^{+}} \delta_{x}(A, B) \text { where } \\
\delta_{x}(A, B) & =\max \left\{\frac{D_{R E}^{A}(x)}{D_{R E}^{B}(x)}, \frac{D_{R E}^{B}(x)}{D_{R E}^{A}(x)}\right\}
\end{aligned}
$$

is called the risk oriented distance between $A$ and $B$.

Lemma 5.4. The following properties are also trivial. Suppose that $\mathfrak{I}_{A}^{+} \cap \mathfrak{I}_{B}^{+} \neq \varnothing$.

i) If $B \geq A$ then

$$
\mathcal{M}_{R E}(A, B)=\sup _{x \in \mathfrak{I}_{A} \cap \mathfrak{I}_{B}} \max \left\{\frac{D_{R E}^{B}(x)}{D_{R E}^{A}(x)}\right\}
$$

ii) If $A=B$ then $\mathcal{M}_{R E}(A, B)=1$.

iii) If $\mathcal{M}_{R E}(A, B)>1$ then $A \neq B$.

This measure is useful to test whether or not the short sales impact the return expansion given some portfolio $x$. In such a case, we have:

$$
\delta_{x}(I, 0)=\max \left\{\frac{D_{R E}^{I}(x)}{D_{R E}^{0}(x)}, \frac{D_{R E}^{0}(x)}{D_{R E}^{I}(x)}\right\},
$$

and since, $\mathfrak{I}_{I} \subset \mathfrak{I}_{0}$, we have

$$
\mathcal{M}_{R E}(I, 0)=\sup _{x \in \mathfrak{I}_{I}} \delta_{x}(I, 0) .
$$

If there exists some portfolio $y \in \mathfrak{J}_{0}$ such that $\rho(y)$ $=\rho(x)$ and $D_{R E}^{I}(x) \mu(x)<\mu(y)$, then it is easy to see that $\mathcal{M}_{R C}(I, 0)>1$. Hence, in this situation, the possibility of short sales enables decision makers to find a riskless portfolio.

In general, it is difficult to compute the map $\mathcal{M}_{R E}(A$, $B)$ and $\mathcal{M}_{R C}(A, B)$. However, one can compute an approximation based upon each specific asset. By construction, we have $R\left(e_{i}\right)=R_{i}$, for $i=1, \cdots, n$ where $\left\{e_{i}\right\}_{i=1, \cdots, n}$ is the canonical basis of $\mathbb{R}^{n}$. This we do by defining

$$
\begin{gathered}
\tilde{\mathcal{M}}_{R C}(I, 0)=\max _{i=1, \cdots, n} d_{e_{i}}(I, 0) \text { and } \\
\tilde{\mathcal{M}}_{R E}(I, 0)=\max _{i=1, \cdots, n} \delta_{e_{i}}(I, 0) .
\end{gathered}
$$

To analyse the case where there are short sales, an approach based upon quadratic programming may be used. However, since the optimization constraints are binding, it is possible to give a solution in closed form for $\gamma^{*}, \beta^{*}$ and $x^{*}$.

\section{Empirical Illustration}

This section presents a numerical example. It is shown that some key contributions of this paper can be easily implemented using standard methods of quadratic programming. The data are obtained from the CAC 40 monthly stocks over a period running from January 1984 to December 2008. For each of the 38 stocks, we have calculated monthly expected returns, covariances, variances with a monthly percentage return. After computing the efficient frontier, we set parameters $\mu=1$ and $\rho=$ 2 . Below, Tables 1 and $\mathbf{2}$ respectively summarize in the risk oriented and return oriented cases the decomposition of monthly performances for each asset. Remember that overall efficiency looks for a global improvement of a title following a chosen direction. Hence, it can be

\begin{tabular}{|c|c|c|c|c|}
\hline Asset & $O_{R C}$ & $D_{R C}$ & $A_{R C}$ & $\gamma_{R C}$ \\
\hline Accor & 2.9767 & 0.0358 & 2.9409 & 0.0000 \\
\hline Air france & 0.8191 & 0.0291 & 0.7900 & 0.0140 \\
\hline Air liquide & 9.6218 & 0.0935 & 9.5283 & 0.0000 \\
\hline Alcaltel & -0.1972 & 0.2086 & -0.4058 & 0.0133 \\
\hline Alstom & -0.6221 & 1.0000 & -1.6221 & 0.0196 \\
\hline Arcelor mittal & 3.6585 & 0.0438 & 3.6147 & 0.0000 \\
\hline Axa & 1.8391 & 0.0225 & 1.8166 & 0.0000 \\
\hline Bnp & 2.5783 & 0.0336 & 2.5447 & 0.0000 \\
\hline Bouygues & 2.6120 & 0.0269 & 2.5851 & 0.0000 \\
\hline Cap gemini & 0.5518 & 0.0147 & 0.5372 & 0.0230 \\
\hline Carrefour & 5.0090 & 0.0489 & 4.9601 & 0.0000 \\
\hline Credit agricole & 2.1384 & 0.1024 & 2.0360 & 0.0097 \\
\hline Danone & 6.1840 & 0.0623 & 6.1218 & 0.0000 \\
\hline Dexia & -0.1622 & 0.3111 & -0.4733 & 0.0128 \\
\hline Eads & 1.2664 & 0.0392 & 1.2272 & 0.0175 \\
\hline Edf & 17.4122 & 0.1721 & 17.2401 & 0.0000 \\
\hline Essilor intl & 9.2642 & 0.0716 & 9.1925 & 0.0000 \\
\hline France telecom & 0.1872 & 0.0333 & 0.1539 & 0.0107 \\
\hline Gdf suez & 41.1744 & 0.3918 & 40.7825 & 0.0000 \\
\hline Lafarge & 2.5799 & 0.0362 & 2.5437 & 0.0000 \\
\hline Lagardere & 2.3142 & 0.0272 & 2.2869 & 0.0000 \\
\hline Loreal & 6.2259 & 0.0522 & 6.1737 & 0.0000 \\
\hline Lvmh & 2.4824 & 0.0286 & 2.4539 & 0.0000 \\
\hline Michelin & 2.6201 & 0.0354 & 2.5847 & 0.0000 \\
\hline Pernod ricard & 6.5931 & 0.0642 & 6.5289 & 0.0000 \\
\hline Peugeot & 1.8569 & 0.0284 & 1.8285 & 0.0000 \\
\hline Ppr & 2.1359 & 0.0258 & 2.1101 & 0.0000 \\
\hline Renault & 0.9004 & 0.0209 & 0.8794 & 0.0319 \\
\hline Saint gobain & 2.7787 & 0.0324 & 2.7463 & 0.0000 \\
\hline Sanofi aventis & 9.4330 & 0.1144 & 9.3185 & 0.0000 \\
\hline Schneider & 3.0940 & 0.0356 & 3.0584 & 0.0000 \\
\hline Societe generale & 2.2684 & 0.0269 & 2.2415 & 0.0000 \\
\hline $\begin{array}{l}\text { Stmicroelec- } \\
\text { tronics }\end{array}$ & 0.9322 & 0.0161 & 0.9161 & 0.1430 \\
\hline Suez E. & 15.3026 & 0.9597 & 14.3429 & 0.0071 \\
\hline Total & 7.2215 & 0.0662 & 7.1553 & 0.0000 \\
\hline Unibail & 5.4427 & 0.0559 & 5.3868 & 0.0000 \\
\hline Vallourec & 2.1729 & 0.0181 & 2.1548 & 0.0000 \\
\hline Veolia environ & 2.1113 & 0.0484 & 2.0628 & 0.0337 \\
\hline Vinci & 3.9696 & 0.0393 & 3.9303 & 0.0000 \\
\hline Vivendi & 1.8895 & 0.0288 & 1.8607 & 0.0000 \\
\hline
\end{tabular}
decomposed in two parts reflecting portfolio efficiency and allocative efficiency.

As it is shown in Table 1, asset 1 (Accor) has a low return and therefore it is too risky. The investor can

Table 1. Decomposition of "CAC 40" performance (Risk Oriented). 
Table 2. Decomposition of "CAC 40" performance (Return Oriented).

\begin{tabular}{|c|c|c|c|c|}
\hline Asset & $O_{R E}$ & $D_{R E}$ & $A_{R E}$ & $\gamma_{R E}$ \\
\hline Accor & 33.6665 & 33.6640 & 0.0025 & 0.0133 \\
\hline Air france & 2.5886 & 2.5561 & 0.0325 & 0.0135 \\
\hline Air liquide & -5.8125 & -5.5131 & -0.2994 & 0.0128 \\
\hline Alcaltel & 1.8827 & 1.5611 & 0.3215 & 0.0170 \\
\hline Alstom & 1.3252 & 1.0000 & 0.3252 & 0.0196 \\
\hline Arcelor mittal & 37.7219 & 37.5822 & 0.1397 & 0.0132 \\
\hline Axa & 27.2942 & 26.9360 & 0.3582 & 0.0135 \\
\hline Bnp & 11.8982 & 11.8983 & -0.0001 & 0.0133 \\
\hline Bouygues & -11.7382 & -11.6888 & -0.0494 & 0.0134 \\
\hline Cap gemini & 3.7739 & 3.4874 & 0.2865 & 0.0142 \\
\hline Carrefour & -6.6492 & -6.5984 & -0.0508 & 0.0132 \\
\hline Credit agricole & 1.7816 & 1.7288 & 0.0528 & 0.0130 \\
\hline Danone & -7.8429 & -7.6831 & -0.1599 & 0.0131 \\
\hline Dexia & 1.3169 & 1.3140 & 0.0030 & 0.0134 \\
\hline Eads & 2.4821 & 2.4815 & 0.0005 & 0.0134 \\
\hline Edf & -6.1239 & -5.4436 & -0.6804 & 0.0122 \\
\hline Essilor intl & -2.3266 & -2.2569 & -0.0697 & 0.0130 \\
\hline France telecom & 2.3786 & 2.1740 & 0.2046 & 0.0147 \\
\hline Gdf suez & -4.6683 & -3.7145 & -0.9538 & 0.0103 \\
\hline Lafarge & 7.5510 & 7.5500 & 0.0010 & 0.0133 \\
\hline Lagardere & 77.9918 & 77.6968 & 0.2950 & 0.0134 \\
\hline Loreal & -3.2041 & -3.1702 & -0.0339 & 0.0132 \\
\hline Lvmh & -307.6488 & -306.9223 & -0.7265 & 0.0134 \\
\hline Michelin & 9.2097 & 9.2095 & 0.0001 & 0.0133 \\
\hline Pernod ricard & -6.2128 & -6.0738 & -0.1390 & 0.0130 \\
\hline Peugeot & 5.7738 & 5.7592 & 0.0146 & 0.0134 \\
\hline Ppr & 33.6970 & 33.4996 & 0.1974 & 0.0134 \\
\hline Renault & 3.4948 & 3.4060 & 0.0888 & 0.0135 \\
\hline Saint gobain & 162.9716 & 162.9394 & 0.0322 & 0.0134 \\
\hline Sanofi aventis & 25.2989 & 23.5278 & 1.7711 & 0.0126 \\
\hline Schneider & -252.3979 & -252.3878 & -0.0100 & 0.0133 \\
\hline $\begin{array}{l}\text { Societe } \\
\text { generale }\end{array}$ & 56.2202 & 55.9829 & 0.2373 & 0.0134 \\
\hline $\begin{array}{l}\text { Stmicroelec- } \\
\text { tronics }\end{array}$ & 5.2064 & 4.9761 & 0.2303 & 0.0136 \\
\hline $\begin{array}{c}\text { Suez } \\
\text { environnement }\end{array}$ & 1.4416 & 1.0284 & 0.4132 & 0.0037 \\
\hline Total & -4.4442 & -4.3357 & -0.1085 & 0.0130 \\
\hline Unibail & -9.4252 & -9.2927 & -0.1325 & 0.0131 \\
\hline Vallourec & -4.2453 & -4.1137 & -0.1315 & 0.0135 \\
\hline Veolia environ & 2.7819 & 2.7700 & 0.0119 & 0.0132 \\
\hline Vinci & -7.6228 & -7.6144 & -0.0085 & 0.0133 \\
\hline Vivendi & 5.8051 & 5.7926 & 0.0125 & 0.0134 \\
\hline
\end{tabular}

choose an efficient portfolio that is $0,036 \%$ less risky and does not require risk-taking $\left(\gamma_{R C}=0\right)$. Considering only risk aversion, it is possible to pursue reduction of risk to maximize the utility of a portfolio but, due to the market imperfections, this result cannot be achieved without short sales.

In the return expansion case, the first asset is less efficient (Table 2) than is the risk oriented case. Given the same level of risk, it should be 50 times more profitable $\left(O_{R E}=33.6665\right)$. However, technically, we can only select a portfolio that produces 33 times more important return $\left(D_{R E}=33.6641\right)$. The market imperfections again limits this return improvement $\left(A_{R E}=0.0025\right)$. Here, the investor must take 6 units of risk to increase his (or her) return of a one unit amount $\left(\gamma_{R E}=0.0133\right)$. Table 3 details implicit risk aversion of risk reduction
Table 3. Implicit Risk Aversion.

\begin{tabular}{|c|c|c|c|c|c|c|}
\hline \multirow[t]{2}{*}{ Asset } & \multicolumn{3}{|c|}{ Risk Oriented } & \multicolumn{3}{|c|}{ Return Oriented } \\
\hline & $\alpha_{R C}$ & $\beta_{R C}$ & $\gamma_{R C}$ & $\alpha_{R E}$ & $\beta_{R E}$ & $\gamma_{R E}$ \\
\hline Accor & 0.000 & 1.000 & 0.000 & 0.006 & 0.482 & 0.013 \\
\hline Air france & 0.000 & 0.011 & 0.014 & 0.008 & 0.613 & 0.013 \\
\hline Air liquide & 0.000 & 1.000 & 0.000 & 0.003 & 0.284 & 0.012 \\
\hline Alcaltel & 0.006 & 0.462 & 0.013 & 0.020 & 1.197 & 0.017 \\
\hline Alstom & 0.030 & 1.534 & 0.019 & 0.030 & 1.534 & 0.019 \\
\hline Arcelor mittal & 0.000 & 1.000 & 0.000 & 0.005 & 0.433 & 0.013 \\
\hline Axa & 0.000 & 1.000 & 0.000 & 0.008 & 0.616 & 0.013 \\
\hline Bnp & 0.000 & 1.000 & 0.000 & 0.006 & 0.499 & 0.013 \\
\hline Bouygues & 0.000 & 1.000 & 0.000 & 0.007 & 0.561 & 0.013 \\
\hline Cap gemini & 0.000 & 0.008 & 0.023 & 0.012 & 0.846 & 0.014 \\
\hline Carrefour & 0.000 & 1.000 & 0.000 & 0.005 & 0.408 & 0.013 \\
\hline Credit agricole & 0.000 & 0.014 & 0.009 & 0.004 & 0.332 & 0.013 \\
\hline Danone & 0.000 & 1.000 & 0.000 & 0.004 & 0.358 & 0.013 \\
\hline Dexia & 0.003 & 0.287 & 0.012 & 0.007 & 0.542 & 0.013 \\
\hline Eads & 0.000 & 0.010 & 0.017 & 0.006 & 0.509 & 0.013 \\
\hline Edf & 0.000 & 1.000 & 0.000 & 0.002 & 0.195 & 0.012 \\
\hline Essilor intl & 0.000 & 1.000 & 0.000 & 0.004 & 0.331 & 0.013 \\
\hline $\begin{array}{l}\text { France } \\
\text { telecom }\end{array}$ & 0.001 & 0.105 & 0.010 & 0.013 & 0.890 & 0.014 \\
\hline Gdf suez & 0.000 & 1.000 & 0.000 & 0.001 & 0.095 & 0.010 \\
\hline Lafarge & 0.000 & 1.000 & 0.000 & 0.006 & 0.480 & 0.013 \\
\hline Lagardere & 0.000 & 1.000 & 0.000 & 0.007 & 0.557 & 0.013 \\
\hline Loreal & 0.000 & 1.000 & 0.000 & 0.005 & 0.394 & 0.013 \\
\hline Lvmh & 0.000 & 1.000 & 0.000 & 0.007 & 0.543 & 0.013 \\
\hline Michelin & 0.000 & 1.000 & 0.000 & 0.006 & 0.485 & 0.013 \\
\hline Pernod ricard & 0.000 & 1.000 & 0.000 & 0.004 & 0.352 & 0.013 \\
\hline Peugeot & 0.000 & 1.000 & 0.000 & 0.007 & 0.545 & 0.013 \\
\hline Ppr & 0.000 & 1.000 & 0.000 & 0.007 & 0.573 & 0.013 \\
\hline Renault & 0.000 & 0.006 & 0.031 & 0.009 & 0.668 & 0.013 \\
\hline Saint gobain & 0.000 & 1.000 & 0.000 & 0.006 & 0.509 & 0.013 \\
\hline Sanofi aventis & 0.000 & 1.000 & 0.000 & 0.003 & 0.252 & 0.012 \\
\hline Schneider & 0.000 & 1.000 & 0.000 & 0.006 & 0.484 & 0.013 \\
\hline $\begin{array}{l}\text { Societe } \\
\text { generale }\end{array}$ & 0.000 & 1.000 & 0.000 & 0.007 & 0.561 & 0.013 \\
\hline $\begin{array}{l}\text { Stmicroelec- } \\
\text { tronics }\end{array}$ & 0.000 & 0.001 & 0.143 & 0.009 & 0.733 & 0.013 \\
\hline Suez E. & 0.000 & 0.017 & 0.007 & 0.000 & 0.021 & 0.003 \\
\hline Total & 0.000 & 1.000 & 0.000 & 0.004 & 0.346 & 0.013 \\
\hline Unibail & 0.000 & 1.000 & 0.000 & 0.005 & 0.380 & 0.013 \\
\hline Vallourec & 0.000 & 1.000 & 0.000 & 0.009 & 0.689 & 0.013 \\
\hline Veolia environ & 0.000 & 0.006 & 0.033 & 0.005 & 0.429 & 0.013 \\
\hline Vinci & 0.000 & 1.000 & 0.000 & 0.006 & 0.459 & 0.013 \\
\hline Vivendi & 0.000 & 1.000 & 0.000 & 0.007 & 0.541 & 0.013 \\
\hline
\end{tabular}

and return expansion for each asset. Note that some assets have a negative performance indicator. This can be explained by the fact that they have a negative return. The portfolio may have a greater risk than their expected returns and their respective utility functions measures the potential loss of each invested euro. This is not consistent with the objective of maximizing behaviour the investors have and, in such a case, allocative efficiency makes no sense.

Improvements both based on increasing expected returns and risk contraction are presented in Table 4. In general, the shortage function and the hyperbolic function give almost the same improvement of performance ( 0.008 for asset 1$)$. The Hyperbolic functions provide a seven times greater improvement than those obtained from these two functions. Hence, much more return, but 
Table 4. "CAC 40" Efficiencies.

\begin{tabular}{|c|c|c|c|c|c|}
\hline Asset & $S_{g}$ & $D_{R C}$ & $D_{R E}$ & $D_{H}$ & $G_{M F}$ \\
\hline Accor & 0.001 & 0.036 & 33.664 & 17.796 & 55.920 \\
\hline Air france & 0.006 & 0.029 & 2.556 & 2.109 & 3.759 \\
\hline Air liquide & 0.001 & 0.093 & -5.513 & 10.691 & \\
\hline Alcaltel & 0.007 & 0.208 & 1.561 & 1.410 & 1.695 \\
\hline Alstom & 0.000 & 1.000 & 1.000 & 1.000 & 1.000 \\
\hline Arcelor mittal & 0.001 & 0.044 & 37.582 & 17.622 & 65.610 \\
\hline Axa & 0.001 & 0.022 & 26.936 & 15.078 & 39.517 \\
\hline Bnp & 0.002 & 0.033 & 11.898 & 7.769 & 19.444 \\
\hline Bouygues & 0.002 & 0.026 & -11.688 & 37.171 & \\
\hline Cap gemini & 0.008 & 0.014 & 3.487 & 2.646 & 4.330 \\
\hline Carrefour & 0.002 & 0.048 & -6.598 & 20.440 & \\
\hline Credit agricole & 0.001 & 0.102 & 1.728 & 1.574 & 2.045 \\
\hline Danone & 0.002 & 0.062 & -7.683 & 16.060 & \\
\hline Dexia & 0.001 & 0.311 & 1.314 & 1.242 & 1.458 \\
\hline Eads & 0.004 & 0.039 & 2.481 & 2.082 & 3.583 \\
\hline Edf & 0.001 & 0.172 & -5.443 & 5.809 & \\
\hline Essilor intl & 0.002 & 0.071 & -2.256 & 13.958 & \\
\hline France telecom & 0.008 & 0.033 & 2.174 & 1.820 & 2.652 \\
\hline Gdf suez & 0.000 & 0.391 & -3.714 & 2.552 & \\
\hline Lafarge & 0.003 & 0.036 & 7.550 & 5.304 & 12.570 \\
\hline Lagardere & 0.000 & 0.027 & 77.696 & 30.111 & 120.149 \\
\hline Loreal & 0.003 & 0.052 & -3.170 & 19.150 & \\
\hline Lvmh & 0.000 & 0.028 & -306.922 & 35.013 & \\
\hline Michelin & 0.002 & 0.035 & 9.209 & 6.271 & 15.251 \\
\hline Pernod ricard & 0.002 & 0.064 & -6.073 & 15.570 & \\
\hline Peugeot & 0.004 & 0.028 & 5.759 & 4.160 & 9.004 \\
\hline Ppr & 0.001 & 0.025 & 33.499 & 18.262 & 51.040 \\
\hline Renault & 0.007 & 0.021 & 3.406 & 2.643 & 4.776 \\
\hline Saint gobain & 0.000 & 0.032 & 162.939 & 30.859 & 263.763 \\
\hline Sanofi aventis & 0.001 & 0.114 & 23.527 & 8.552 & 50.616 \\
\hline Schneider & 0.000 & 0.035 & -252.387 & 28.082 & \\
\hline $\begin{array}{c}\text { Societe } \\
\text { generale }\end{array}$ & 0.001 & 0.027 & 55.983 & 25.723 & 86.276 \\
\hline $\begin{array}{l}\text { Stmicroelec- } \\
\text { tronics }\end{array}$ & 0.006 & 0.016 & 4.976 & 3.564 & 6.620 \\
\hline Suez E. & 0.000 & 0.959 & 1.028 & 1.017 & 1.057 \\
\hline Total & 0.003 & 0.066 & -4.335 & 15.103 & \\
\hline Unibail & 0.002 & 0.056 & -9.293 & 17.886 & \\
\hline Vallourec & 0.007 & 0.018 & -4.114 & 55.354 & \\
\hline Veolia environ & 0.004 & 0.048 & 2.770 & 2.306 & 4.102 \\
\hline Vinci & 0.003 & 0.039 & -7.614 & 25.461 & \\
\hline Vivendi & 0.004 & 0.029 & 5.793 & 4.184 & 9.091 \\
\hline
\end{tabular}

also much more risk (56 more times for asset 1) are involved with the McFadden Gauge [4].

Intuitively, this last measure may be use for a risklover manager. The mean return expansion function is suitable for risk-neutral investor. Risk averse manager may be appreciate by risk contraction, hyperbolic or shortage functions.

Finally, regarding to all the results calculated in Table 4, il clearly appears that the efficient frontier is entirely characterized by two funds: Asset 5 (Alsthom) and Asset 34 (Suez environment). This is an illustration, of the two funds theorem.

\section{Conclusions}

This paper has analyzed duality relations between the indirect mean-variance utility function and a broad class of portfolio efficiency measures. It has been shown that such approaches are useful to measure the impact of managerial constraints on the performance. In addition, the implicit risk aversion of an optimal solution can be deduced from the Kuhn-Tucker optimality conditions.

\section{References}

[1] M. R. Morey and R. C. Morey, "Mutual Fund Performance Appraisals: A Multi-horizon Perspective with Endogenous Benchmarking," Omega, Vol. 27, No. 2, 1999, pp. 241-258. doi:10.1016/S0305-0483(98)00043-7

[2] W. Briec, K. Kerstens and J.-B. Lesourd, "Single Period Markowitz Portfolio Selection, Performance Gauging and Duality: A Variation on Luenberger's Shortage Function," Journal of Optimization Theory and Applications, Vol. 120, No. 1, 2004, pp. 1-27. doi:10.1023/B:JOTA.0000012730.36740.bb

[3] W. Briec, K. Kerstens and O. Jokung, "Mean-VarianceSkewness Portfolio Performance Gauging: A General Shortage Function and Dual Approach," Management Science, Vol. 53, No. 1, 2007, pp. 135-149. doi: $10.1287 / \mathrm{mnsc} .1060 .0596$

[4] D. McFadden, "Cost, Revenue, and Profit Functions," In: M. Fuss and D. McFadden, Eds., Production Economics: A Dual Approach to Theory and Applications, Amsterdam, North-Holland, Vol. 1, 1978, pp. 3-109.

[5] R. W. Shephard, "Cost and Production Functions," Princeton University Press, Princeton, 1953.

[6] H. Markowitz, "Portfolio Selection," Journal of Finance, Vol. 7, No. 1, 1952, pp. 77-91. doi: $10.2307 / 2975974$

[7] H. Markowitz, "Portfolio Selection: Efficient Diversification of Investments," John Wiley, New York, 1959.

[8] W. Sharpe, "A Simplified Model for Portfolio Analysis," Management Science, Vol. 9, No. 2, 1963, pp. 277-293. doi: $10.1287 / \mathrm{mnsc}$.9.2.277

[9] W. Sharpe, "Capital Asset Prices: A Theory of Market Equilibrium under Condition of Risk," Journal of Finance, Vol. 19, No. 3, 1964, pp. 425-442. doi: $10.2307 / 2977928$

[10] J. Lintner, "The Valuation of Risk Assets and the Selection of Risky Investment in Stock Portfolios and Capital Budgets," Review of Economics and Statistics, Vol. 47, No. 1, 1965, pp. 13-37. doi:10.2307/1924119

[11] H. Markowitz, "CAPM Investors do Not Get Paid for Bearing Risk: A Linear Relation does Not Imply Payment for Risk," Journal of Portfolio Management, Vol. 34, No. 2, 2008, pp. 91-96.

[12] D. G. Luenberger, "Microeconomic Theory," McGraw Hill, Boston, 1995.

[13] M. Farrell, "The Measurement of Productive Efficiency," Journal of the Royal Statistical Society, Vol. 120, No. 3, 1957, pp. 253-281. doi: $10.2307 / 2343100$

[14] J. A. Pogue, "An Extension of the Markowitz Portfolio Selection Model to include Variable Transaction Cost, 
short Sales, Leverage Polycies and Taxes," Journal of Finance, Vol. 25, No. 5, 1970, pp. 1005-1028. doi: $10.2307 / 2325576$

[15] A. Rudd and B. Rosenberg, "A Realistic Portfolio Selection Model," In: E. J. Elton and M. J. Grubber, Eds., Portfolio Theory-Lectures in Management Science, Vol. II, North Holland, Amsterdam, 1979, pp. 21-46.

[16] A. Charnes, W. W. Cooper and E. Rhodes, "Measuring the Efficiency of Decision-Making Units," European Journal of Operational Research, Vol. 3, No. 6, 1978, pp. 429-444. doi:10.1016/0377-2217(78)90138-8

[17] J. K. Sengupta, "Nonparametric Tests of Efficiency of Portfolio Investment," Journal of Economics, Vol. 50, No. 1, 1989, pp. 1-15. doi:10.1007/BF01227605

\section{Appendix: The Short Sale Case Computations}

It is possible to give a solution of the case where there are short sales with an approach based upon quadratic programming.

\section{Computation of Risk Oriented Measure}

In the following, we assume that $\mu(x) \geq \mu\left(x^{\#}\right)$ where $x^{\#} \in \operatorname{argmin}\left\{\rho(x): x \in \mathfrak{I}_{0}\right\}$. Under such an assumption, we know that $\left(D_{R C}^{0}(x) \rho(x), \mu(x)\right)$ lies on the curve representing the hyperbola relating the expected returns and the variance. We first write the Lagrangian of the optimization problem we need to solve. It is defined by

$$
\begin{aligned}
& L(x, \lambda, \alpha, \beta, \gamma)=\lambda-\alpha\left(\sum_{i} x_{i} E\left[R_{i}\right]-E\left[R_{p}\right]\right) \\
& -\beta\left(\lambda \operatorname{Var}\left[R_{p}\right]-\sum_{i, j} x_{i} x_{j} \Omega_{i, j}\right)-\gamma\left(\sum_{i} x_{i}-1\right)
\end{aligned}
$$

Suppose that $\left(x^{*}, \alpha^{*}, \beta^{*}, \gamma^{*}, \lambda^{*}\right)$ is solution. The first order conditions yield:

$$
\begin{gathered}
\frac{\partial}{\partial x_{i}} L(x, \delta, \alpha, \beta, \gamma)=-\alpha E\left[R_{i}\right]+\beta \sum_{j} x_{j} \Omega_{i, j}-\gamma=0 \\
\frac{\partial}{\partial \lambda} L(x, \delta, \alpha, \beta, \gamma)=1-\beta \operatorname{Var}\left[R_{p}\right]=0
\end{gathered}
$$

Following (7.2); we have
[18] R. Färe, S. Grosskopf, C. A. K. Lovell, "The Measurement of Efficiency of Production," Kluwer, Boston, 1985.

[19] W. Briec and J.-B. Lesourd, "The Efficiency of Investment Fund Management: A Stochastic Frontier Model," In: Dunis, Eds., Advances in Quantitative Asset Management, Klwer Academic Publishers, Norwell. 2000, pp. 41-58.

[20] A. Ruszczynski and R. Vanderbei, "Frontiers of Stochastically Nondominated Portfolios," Econometrica, Vol. 71, No. 4, 2003, pp. 1287-1297. doi:10.1111/1468-0262.t01-1-00448

[21] A. V. Fiacco and G. P. McGormick, "Nonlinear Programming: Sequential Uncontrained Minimization Techniques," John Wiley, New York, 1968.

$$
x=\boldsymbol{\Omega}^{-1}\left(\frac{\gamma}{\beta} 1_{n}+\frac{\alpha}{\beta} E[R]\right)
$$

Since $E[R(x)] \geq E\left[R\left(x^{\#}\right)\right]$ the constraints are binding. Hence, substituting in the first order conditions, we have a three-dimensional system to solve, with:

$$
\begin{gathered}
{ }^{t} E[R]\left(\frac{\gamma}{\beta} 1_{n}+\frac{\alpha}{\beta} E[R]\right)-E\left[R_{p}\right]=0 \\
{ }^{t}\left(\frac{\gamma}{\beta} 1_{n}+\frac{\alpha}{\beta} E[R]\right) \boldsymbol{\Omega}^{-1}\left(\frac{\gamma}{\beta} 1_{n}+\frac{\alpha}{\beta} E[R]\right)-\lambda V\left[R_{p}\right]=0 \\
{ }^{t} 1_{n} \mathbf{\Omega}^{-1}\left(\frac{\gamma}{\beta} 1_{n}+\frac{\alpha}{\beta} E[R]\right)=1
\end{gathered}
$$

The last equation yields:

$$
\frac{\gamma}{\beta}=\left(\frac{1}{{ }^{t} 1_{n} \mathbf{\Omega}^{-1} 1_{n}}\right)-\frac{\alpha}{\beta}\left({ }^{t} 1_{n} \boldsymbol{\Omega}^{-1}\right) E[R]
$$

Substituting in 7.5 , we obtain:

$$
\begin{array}{r}
{ }^{t} E[R]\left[\left(\frac{1}{{ }^{t} 1_{n} \mathbf{\Omega}^{-1} 1_{n}}\right) 1_{n}-\frac{\alpha}{\beta}\left({ }^{t} 1_{n} \boldsymbol{\Omega}^{-1}\right) E[R] 1_{n}\right. \\
\left.+\frac{\alpha}{\beta}\left(\mathbf{\Omega}^{-1}\right) E[R]\right]-E\left[R_{p}\right]=0 .
\end{array}
$$

Hence, we obtain the optimal values: 


$$
\begin{aligned}
\frac{\alpha^{*}}{\beta^{*}}= & {\left[{ }^{t} E[R]\left({ }^{t} 1_{n} \boldsymbol{\Omega}^{-1}\right) E[R] 1_{n}-{ }^{t} E[R] \boldsymbol{\Omega}^{-1} E[R]\right]^{-1} } \\
& \times\left[{ }^{t} E[R]\left(\frac{1}{{ }^{t} 1_{n} \boldsymbol{\Omega}^{-1} 1_{n}}\right) 1_{n}+E\left[R_{p}\right]\right]
\end{aligned}
$$

and $\gamma^{*}$ is then obtained from equation 46. From 52, we deduce $x^{*}$. The distance function is given by $\lambda^{*}$

$$
\begin{aligned}
& \lambda^{*}=\operatorname{Var}\left[R_{p}\right]^{-1} \times\left[\left(\frac{1}{{ }^{t} 1_{n} \mathbf{\Omega}^{-1} 1_{n}}\right) 1_{n}\right. \\
& \left.-\frac{\alpha^{*}}{\beta^{*}}\left({ }^{t} 1_{n} \boldsymbol{\Omega}^{-1}\right) E[R] 1_{n}+\frac{\alpha^{*}}{\beta^{*}} \boldsymbol{\Omega}^{-1} E[R]\right] \\
& \times \mathbf{\Omega}\left[\left(\frac{1}{{ }^{t} 1_{n} \boldsymbol{\Omega}^{-1} 1_{n}}\right) 1_{n}-\frac{\alpha^{*}}{\beta^{*}}\left({ }^{t} 1_{n} \boldsymbol{\Omega}^{-1}\right) E[R] 1_{n}\right. \\
& \left.+\frac{\alpha^{*}}{\beta^{*}} \boldsymbol{\Omega}^{-1} E[R]\right]
\end{aligned}
$$

\section{Computation of Return Oriented Measure}

In the following, we suppose that $E\left[R_{p}\right]>0$. The Lagrangian of the optimization problem we need to optimize is:

$$
\begin{array}{r}
L(x, \lambda, \alpha, \beta, \gamma)=-\theta-\alpha\left(\sum_{i} x_{i} E\left[R_{i}\right]-\theta E\left[R_{p}\right]\right) \\
-\beta\left(\operatorname{Var}\left[R_{p}\right]-\sum_{i, j} x_{i} x_{j} \Omega_{i, j}\right)-\gamma\left(\sum_{i} x_{i}-1\right)
\end{array}
$$

Suppose that $\left(x^{*}, \alpha^{*}, \beta^{*}, \gamma^{*}, \lambda^{*}\right)$ is solution. The first order conditions yield:

$$
\begin{gathered}
\frac{\partial}{\partial x_{i}} L(x, \delta, \alpha, \beta, \gamma)=-\alpha E\left[R_{i}\right]+\beta \sum_{j} x_{j} \Omega_{i, j}-\gamma=0 \\
\frac{\partial}{\partial x_{i}} L(x, \delta, \alpha, \beta, \gamma)=-\alpha E\left[R_{i}\right]=0 \\
\frac{\partial}{\partial \theta} L(x, \delta, \alpha, \beta, \gamma)=1-\alpha E\left[R_{p}\right]=0
\end{gathered}
$$

At the optimum, we clearly have $\alpha^{*}=1 / E\left[R_{p}\right]$. Following (7.2); we have

$$
x=\boldsymbol{\Omega}^{-1}\left(\frac{\gamma}{\beta} 1_{n}+\frac{\alpha}{\beta} E[R]\right)
$$

Since the constraints are binding, substituting in the first order conditions, we have a three-dimensional system to solve, with:

$$
\begin{gathered}
{ }^{t} E[R]\left(\frac{\gamma}{\beta} 1_{n}+\frac{\alpha}{\beta} E[R]\right)-\theta E\left[R_{p}\right]=0 \\
{ }^{t}\left(\frac{\gamma}{\beta} 1_{n}+\frac{\alpha}{\beta} E[R]\right) \boldsymbol{\Omega}^{-1}\left(\frac{\gamma}{\beta} 1_{n}+\frac{\alpha}{\beta} E[R]\right) \\
-\operatorname{Var}\left[R_{p}\right]=0 \\
{ }^{t} 1_{n} \mathbf{\Omega}^{-1}\left(\frac{\gamma}{\beta} 1_{n}+\frac{\alpha}{\beta} E[R]\right)=1
\end{gathered}
$$

Combining 7.15 and 7.16 we obtain

$$
{ }^{t}\left(\theta E\left[R_{p}\right]\right) \boldsymbol{\Omega}^{-1}\left(\theta E\left[R_{p}\right]\right)-\|E[R]\|^{2} \operatorname{Var}\left[R_{p}\right]=0
$$

It follows that

$$
\begin{gathered}
\theta^{*}=\left[{ }^{t} E\left[R_{p}\right] \boldsymbol{\Omega}^{-1 / 2} E\left[R_{p}\right]\right]^{-1 / 2} \\
\|E[R]\|\left[\operatorname{Var}\left[R_{p}\right]\right]^{1 / 2}
\end{gathered}
$$

Using 7.17 and 7.15 we obtain:

$$
\begin{array}{r}
{ }^{t} E[R]\left[\left(\frac{1}{{ }^{t} 1_{n} \boldsymbol{\Omega}^{-1} 1_{n}}\right) 1_{n}-\frac{\alpha}{\beta}\left({ }^{t} 1_{n} \boldsymbol{\Omega}^{-1}\right) E[R] 1_{n}+\right. \\
\left.\frac{\alpha}{\beta}\left(\boldsymbol{\Omega}^{-1}\right) E[R]\right]-\theta^{*} E\left[R_{p}\right]=0
\end{array}
$$

Hence, we obtain the optimal values:

$$
\begin{aligned}
\frac{\alpha^{*}}{\beta^{*}}= & {\left[{ }^{t} E[R]\left({ }^{t} 1_{n} \boldsymbol{\Omega}^{-1}\right) E[R] 1_{n}-{ }^{t} E[R] \boldsymbol{\Omega}^{-1} E[R]\right]^{-1} } \\
& \times\left[{ }^{t} E[R]\left(\frac{1}{{ }^{t} 1_{n} \boldsymbol{\Omega}^{-1} 1_{n}}\right) 1_{n}+\theta^{*} E\left[R_{p}\right]\right] .
\end{aligned}
$$

Therefore $\gamma^{*}$ and $\beta^{*}$ are then obtained from equation 7.20. From 7.14, we deduce $x^{*}$. 